\title{
The Clinical Significance of a 'Crazy-Paving' Pattern on Chest Radiology
}

\author{
Ahmed Ehab, Radu I. Braga \\ Pulmonary Department, Loewenstein Lung Center, Löwenstein, Germany
}

Received: $10 / 11 / 2018$

Accepted: $23 / 11 / 2018$

Published: $14 / 12 / 2018$

How to cite this article: Ehab A, Braga RI. The clinical significance of a 'crazy-paving' pattern on chest radiology. EJCRIM 2018;5: doi:10.12890/2018_000978.

Conflicts of Interests: The Authors declare that there are no competing interests.

This article is licensed under a Commons Attribution Non-Commercial 4.0 License

\section{ABSTRACT}

A crazy-paving pattern is a non-specific radiological sign which is characterized by the presence of diffuse ground? glass attenuation associated with interlobular septal thickening and intralobular lines. It was initially described as a pathognomonic sign of pulmonary alveolar proteinosis. However, it can be also found in many other diffuse acute and chronic lung diseases including diffuse alveolar haemorrhage (DAH), a rare and life-threatening clinical syndrome which can be caused by many conditions, the most frequent of these being capillaritis associated with systemic autoimmune diseases.

In this case report, we describe an 82-year-old female patient with acute respiratory failure and bilateral pulmonary infiltrates with the characteristic crazy-paving pattern. The final diagnosis was isolated DAH induced by microscopic polyangiitis. The patient was treated with IV high dose prednisolone and cyclophosphamide and was mechanically ventilated. Nevertheless, her clinical status progressively deteriorated and she died after 3 days from acute respiratory distress syndrome.

\section{LEARNING POINTS}

- A crazy-paving pattern is a non-specific radiological sign which is characterized by the presence of diffuse ground-glass attenuation associated with interlobular septal thickening.

- The presence of a crazy-paving pattern together with a progressive fall in haemoglobin levels suggests diffuse alveolar haemorrhage (DAH).

- A panel for collagen vascular diseases and vasculitis should be immediately performed in patients suspected of having DAH.

\section{KEYWORDS}

Crazy paving, diffuse alveolar hemorrhage, microscopic polyangiitis

\section{CASE DESCRIPTION}

An 82-year-old woman without a known previous history of pulmonary diseases was transferred to our hospital for further investigation and treatment of acute respiratory failure with bilateral pulmonary infiltrates on chest imaging. Her complaints had started 2 weeks previously with fever, generalized bone aches and no obvious cough. The patient was first treated for influenza. However, due to elevated CRP and WBC values, she was then treated for bilateral pneumonia. Blood tests also revealed a progressive fall in haemoglobin levels and elevated anti-MPO antibodies (p-ANCA). A high-resolution CT scan of the chest showed a crazy-paving pattern (Fig. 1).

Based upon the radiological findings, the progressive fall in haemoglobin levels and the results of bronchoalveolar lavage, the diagnosis of microscopic polyangiitis (MPA)-induced diffuse alveolar haemorrhage (DAH) was confirmed. However, despite IV treatment with highdose prednisolone and cyclophosphamide, the patient's clinical status progressively deteriorated, so invasive mechanical ventilation was initiated. Unfortunately, the patient died after 3 days from acute respiratory distress syndrome. 


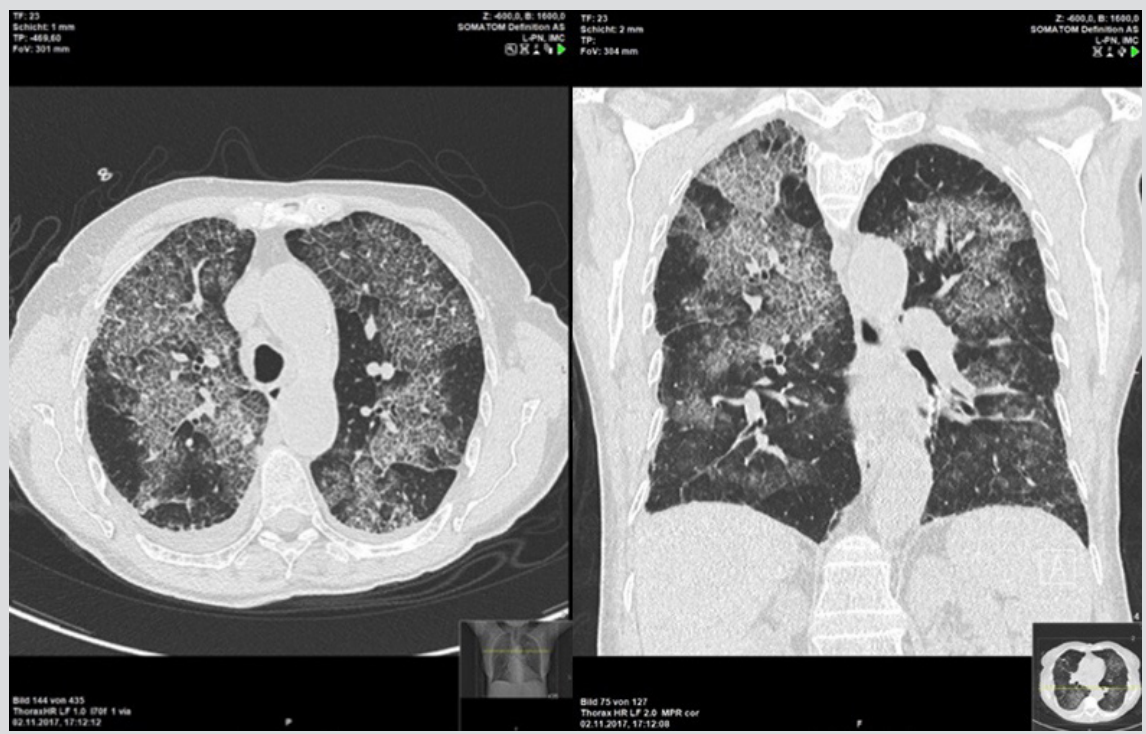

Figure 1. CT of the chest showing a crazy-paving pattern in a patient with microscopic polyangiitisinduced isolated diffuse alveolar haemorrhage

\section{DISCUSSION}

MPA is a pauci-immune, ANCA-associated necrotizing vasculitis that typically affects small blood vessels with histological absence of granuloma $^{[1,2]}$. It is one of the pulmonary-renal vasculitis syndromes that causes DAH in the lungs (more frequently affected) and acute kidney injury (AKI) in the kidneys ${ }^{[1]}$. The incidence of MPA is about 1 in 100,000 per year, with a slight male predominance and a mean age at onset of approximately 50 years ${ }^{[2,3]}$. DAH occurs in $11 \%$ of patients with MPA associated with extrathoracic involvement. Isolated DAH, as described in our case, is very rare ${ }^{[3]}$. The absence of haemoptysis does not exclude the presence of DAH. This can be explained by the large surface area of the alveoli which can absorb a considerable amount of fluid, thus stopping blood reaching the proximal airways ${ }^{[3]}$.

\section{REFERENCES}

1. Segraves JM, lyer VN. Microscopic polyangiitis: atypical presentation with extensive small bowel necrosis, diffuse alveolar hemorrhage, and renal failure. Respir Med Case Rep 2017;21:12-15.

2. Lababidi MH, Odigwe C, Okolo C, Elhassan A. Microscopic polyangiitis causing diffuse alveolar hemorrhage and rapidly progressive glomerulonephritis. Proc (Bayl Univ Med Cent) 2015;28:469-471.

3. Collins CE, Quismorio FP Jr. Pulmonary involvement in microscopic polyangiitis. Curr Opin Pulm Med 2005;11:447-452. 